\title{
Pulmonary DWCNT Exposure Causes Sustained Local and Low- Level Systemic Inflammatory Changes in Mice
}

\author{
Furong Tian \\ Technological University Dublin, furong.tian@tudublin.ie \\ Junja C. Habel-Ungewitter \\ Helmholtz Zentrum Munchen \\ Yin Renfu \\ Jilin University
}

See next page for additional authors

Follow this and additional works at: https://arrow.tudublin.ie/nanolart

Part of the Medicine and Health Sciences Commons

\section{Recommended Citation}

Tian, Furong., Habel-Ungewitter, Nunja. \& Renfu, Y. (2013). Pulmonary DWCNT exposure causes sustained local and low-level systemic inflammatory changes in mice. European Journal of Pharmaceutics and Biopharmaceutics, vol. 84, no. 2, pp. 412-420. doi:10.1016/j.ejpb.2013.03.008.

This Article is brought to you for free and open access by the NanoLab at ARROW@TU Dublin. It has been accepted for inclusion in Articles by an authorized administrator of ARROW@TU Dublin. For more information, please contact arrow.admin@tudublin.ie, aisling.coyne@tudublin.ie,gerard.connolly@tudublin.ie.

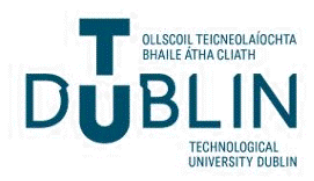




\section{Authors}

Furong Tian, Junja C. Habel-Ungewitter, Yin Renfu, and Tobias Stoger

This article is available at ARROW@TU Dublin: https://arrow.tudublin.ie/nanolart/67 
Research paper

\title{
Pulmonary DWCNT exposure causes sustained local and low-level systemic inflammatory changes in mice
}

\author{
Furong Tian ${ }^{\mathrm{a}}$, Nunja C. Habel ${ }^{\mathrm{a}}$, Renfu Yin ${ }^{\mathrm{a}}$, Stephanie Hirn ${ }^{\mathrm{a}}$, Atrayee Banerjee ${ }^{\mathrm{b}}$, Nuran Ercal ${ }^{\mathrm{b}}$, \\ Shinji Takenaka ${ }^{a}$, Giovani Estrada ${ }^{c}$, Kostas Kostarelos ${ }^{d}$, Wolfgang Kreyling ${ }^{a}$, Tobias Stoeger ${ }^{a, *}$ \\ ${ }^{a}$ Comprehensive Pneumology Center, Institute of Lung Biology and Disease, Helmholtz Zentrum München, Germany \\ ${ }^{\mathrm{b}}$ Department of Chemistry, Missouri University of Science and Technology, Rolla, MO, USA \\ ${ }^{\mathrm{c}}$ Institute of Bioinformatics, Helmholtz Zentrum München, Germany \\ ${ }^{\mathrm{d}}$ Nanomedicine Laboratory, Centre for Drug Delivery Research, UCL School of Pharmacy, University College London, United Kingdom
}

\section{A R T I C L E I N F O}

\section{Article history:}

Available online 28 March 2013

\section{Keywords:}

Carbon nanotubes

DWCNT

Cytokines

Glutathione

Pulmonary inflammation

Systemic inflammation

Phagocytosis

\begin{abstract}
A B S T R A C T
Carbon nanotubes (CNTs) represent promising vectors to facilitate cellular drug delivery and to overcome biological barriers, but some types may also elicit persistent pulmonary inflammation based on their fibre characteristics. Here, we show the pulmonary response to aqueous suspensions of block copolymer dispersed, double-walled carbon nanotubes (DWCNT, length $1-10 \mu \mathrm{m}$ ) in mice by bronchoalveolar lavage (BAL) analysis, and BAL and blood cytokine and lung antioxidant profiling. The intratracheally instilled dose of $50 \mu \mathrm{g}$ DWCNT caused significant pulmonary inflammation that was not resolved during a 7day observation period. Light microscopy investigation of the uptake of DWCNT agglomerates revealed no particle ingestion for granulocytes, but only for macrophages. Accumulating macrophage, multinucleated macrophage and lymphocyte numbers in the alveolar region further indicated ineffective resolution with chronification of the inflammation. The local inflammatory impairment of the lung was accompanied by pulmonary antioxidant depletion and haematological signs of systemic inflammation. While the observed inflammation during its acute phase was dominated by neutrophils and neutrophil recruiting cytokines, the contribution of macrophages and lymphocytes with related cytokines became more significant after day 3 of exposure. This study confirms that acute pulmonary toxicity can occur on exposure of high doses of DWCNT agglomerates and offers further insight for improved nanotube design parameters to avoid potential long-term toxicity.
\end{abstract}

(c) 2013 Elsevier B.V. All rights reserved.

\section{Introduction}

A large number of engineered nanomaterials are currently being investigated for their potential use in biomedical applications such as diagnostics and drug delivery. Due to their one-dimensional nanostructure, carbon nanotubes (CNTs) have shown promise to facilitate cellular delivery and to overcome biological barriers [1]. In particular, single wall carbon nanotubes (SWCNTs), which consist of only one carbon layer and thereby feature the smallest tube diameter of only $1-2 \mathrm{~nm}$, seem advantageous for drug delivery to intracellular targets, since these CNT specimens may enter the cells

\footnotetext{
* Corresponding author. Comprehensive Pneumology Center, Institute of Lung Biology and Disease, Helmholtz Zentrum München, Germany. Tel.: +49 893187 3104; fax: +49 8931872400.

E-mail addresses: furong.tian@gmail.com (F. Tian), nunja.habel@helmholtzmuenchen.de (N.C. Habel), renfu.yin@helmholtz-muenchen.de (R. Yin), stephanie. hirn@med.uni-muenchen.de (S. Hirn), banerjeeat@mst.edu (A. Banerjee), nercal@ mst.edu (N. Ercal), takenaka@helmholtz-muenchen.de (S. Takenaka), giovani. estrada@helmholtz-muenchen.de (G. Estrada), k.kostarelos@ucl.ac.uk (K. Kostarelos), tobias.stoeger@helmholtz-muenchen.de (T. Stoeger).
}

by non-specific, physical penetration of the cell membrane without cytotoxic response [2-4]. On the other hand, the pharmaceutical application of SWCNT is limited due to difficulties in covalent surface functionalization without causing collateral damage to the carbon backbone and thereby changing the tube morphology and surface chemistry. The use of multi-walled (MWCNT) or already double walled (DWCNT) carbon nanotubes could however avoid these pitfalls [5]. Furthermore, DWCNT which have almost the same outer diameter as SWCNT are not as rigid as MWCNT and might therefore, just as SWCNT, be considered less toxic [6].

Besides these promising features, these new fibre materials have raised concerns regarding potential pathogenic effects when they gain access to the lung due to their asbestos-like appearance [7-9] and therefore require individual testing. Independent of particle shape, pulmonary exposure to sufficient doses of carbonaceous nanoparticles has been shown to trigger acute inflammatory effects on the lungs dependent on the total particle surface area, which might resolve after a few days [10-12]. Once aerosolized and airborne, one of the most hazardous exposure routes is the respiratory tract, where inhaled sub- $100 \mathrm{~nm}$ particles may 
deposit in the periphery of the lungs and biopersist by insufficient clearance [13]. Accordingly, there is also an increasing number of reports on potentially adverse health effects of inhaled engineered nanomaterials $[8,14-16]$. Interactions of CNTs with the lung tissue have been reported to induce pro-inflammatory and even pro-fibrotic responses $[17,18]$. The needle-like shape of nanofibres is believed to account for any long-term toxicity of certain types of these innovative, elongated nanomaterials [19,20]. Given the known health impact of mineral fibres (especially the fibrogenic and carcinogenic risk of asbestos fibres), toxicological comparisons of asbestos with fibre-like CNTs (in terms of the elongated shape) have been carried out to assess whether certain CNTs pose a similar health risk $[9,11]$. As a result, a threshold length of approximately $5 \mu \mathrm{m}$ was recently suggested for nanofibre-induced acute pleural inflammation in mice [21], while a threshold CNT length exceeding $15 \mu \mathrm{m}$ has been suggested to increase their toxic risk based on frustrated phagocytosis studies [22,23]. Determining the fibre length, however inside the lungs, remains challenging as well as whether individual tubes or even agglomerated clusters of tubes building up fibres should be considered is currently under discussion.

Besides shape, surface chemistry and purity of CNTs is a subject of intense research [14]. In this context several reports have shown that pristine, non-chemically functionalized CNTs can induce both inflammatory and fibrotic reactions, either upon aspiration instillation or inhalation exposure [9,24-26]. A variety of nanotube types, such as single-wall carbon nanotubes (SWCNTs) and multi-walled carbon nanotubes (MWCNTs), are currently being extensively studied, but no clear general conclusion can be drawn so far regarding contributions of the number of carbon layers to CNT toxicity. Accordingly, a recent study concluded that certain types of MWCNTs can induce mesothelioma and lung cancer, as much as asbestos fibres do, due to their length and high rigidity [27]. Regarding the effect of chemical surface functionalization of nanotubes, even though significant improvement is obtained compared to non-functionalized surfaces, more data are needed to determine the contribution of the types of chemical modifications to their overall toxicological profile.

Double-walled carbon nanotubes (DWCNTs) are rather exceptional representatives of CNTs and might be rated as posing similar toxic risks to that of SWCNTs. Upon agglomeration, SWCNTs form low density, wool-like clumped structures (but not necessarily needle-like structures) as have been described for some types of rigid MWCNTs [19]. For biomedical applications, functionalized CNTs have emerged as a new family of nanovectors $[1,28]$ and DWCNTs as well as MWCNTs are preferred over SWCNTs for covalent functionalization [5]. Even if short $(<10 \mu \mathrm{m})$ and flexible DWCNTs raise less concerns regarding fibre-toxicity characteristics, their safety for pulmonary applications must still be validated in detail.

Here, we explored the acute pulmonary response of intratracheally instilled pristine, block-copolymer dispersed DWCNTs in lungs of young and healthy mice. Since inflammation is known to be the major acute effect of pulmonary particle deposition, we focused on the analysis of inflammatory cells and cytokines, locally in the alveolar compartment of the lung, and systemically in the blood and serum of mice, from 1 to 7 days after exposure. We further investigated particle uptake and antioxidant depletion in lung tissue. Our examinations showed that a dose of $50 \mu \mathrm{g}$ of DWCNT elicits significant, inflammatory responses in the lungs and the blood, which does not resolve within 7 days after treatment.

\section{Methods}

Female BALB/cAnNCrl mice (Charles River Laboratories, Sulzfeld, Germany), at the age of 10-12 weeks, were exposed to
$50 \mu \mathrm{g}$ pristine DWCNT suspensions in a volume of $50 \mu$ l vehicle by intratracheal instillation as described earlier [11]. According to the data sheet, DWCNTs (Nanocyl, Belgium; NC2100 series) are characterised dimensions of $3.5 \mathrm{~nm}$ in diameter and between 1 and $10 \mu \mathrm{m}$ in length, and carbon purity of over $90 \%$ and metal oxide impurities below 10\%. ICPMS analysis revealed $2.45 \% \mathrm{Fe}$ and $177 \mathrm{mg} / \mathrm{kg}$ Ni impurities in the DWCNT samples with $\mathrm{Cd}, \mathrm{Cr}$, $\mathrm{Cu}$ and $\mathrm{Zn}$ below the detection limits of $8.18 ; 12.7 ; 26.8$ and $38.2 \mathrm{mg} / \mathrm{kg}$, respectively. The specific surface area (BET) was $660 \mathrm{~cm}^{2} / \mathrm{g}$. The absence of any bioactive endotoxin contamination was approved by LIMULUS assay and even more relevant by in vitro studies with the LPS sensitive alveolar macrophage cell line MHS. Since suspensions of pristine DWCNT tend to rapidly form agglomerates in pure water and are thus not stable for instillation application, we used the non-ionic surface-active polyol, Pluronic ${ }^{\circledR}$ F127 (Sigma-Aldrich, Germany) for dispersion. DWCNT were dispersed in pyrogen free, distilled water with $1 \%$ Pluronic F-127, sonicated for 30 min and then further diluted $1: 10$ in water with $1 \% \mathrm{~F}$ 127. Fig. 1 shows a transmission electron microscopy image of the dispersed DWCNT sample. Particle suspensions were stable for several minutes (Z-Average: $454 \mathrm{~nm}$, PdI: 0.7; further details are described elsewhere) and were kept in an ultrasonic water bath and thoroughly mixed by vortexing just prior to instillation. Sham groups received $50 \mu$ vehicle ( $1 \%$ Pluronic F-127 solution). Animals were treated humanely, and with regard to alleviation of suffering, experimental protocols were reviewed and approved by the Bavarian Animal Research Authority.

A total number of 30 mice where used, 8 for control, 5 for sham, 6 for day 1, 6 for day 3 and 5 for day 7. Mice were sacrificed 1, 3 or 7 days post-instillation with an overdose of ketamine/xylazin (1\%/ $0.1 \%$ ) by exsanguination. Blood was drawn from the retro-orbital plexus and collected in EDTA tubes (Sarstedt) for haematological analysis using the ADVIA120 haematology system (Bayer Diagnostics) and non-EDTA-covered tubes to gain serum for later cytokine analysis. The lungs of the mice were lavaged with PBS solution $\left(37^{\circ} \mathrm{C}\right)$, and bronchoalveolar lavage (BAL) was processed, as previously described [11]. For each mouse, four lavage cell cytospins were prepared; two used for cell differentiation by May-Grünwald

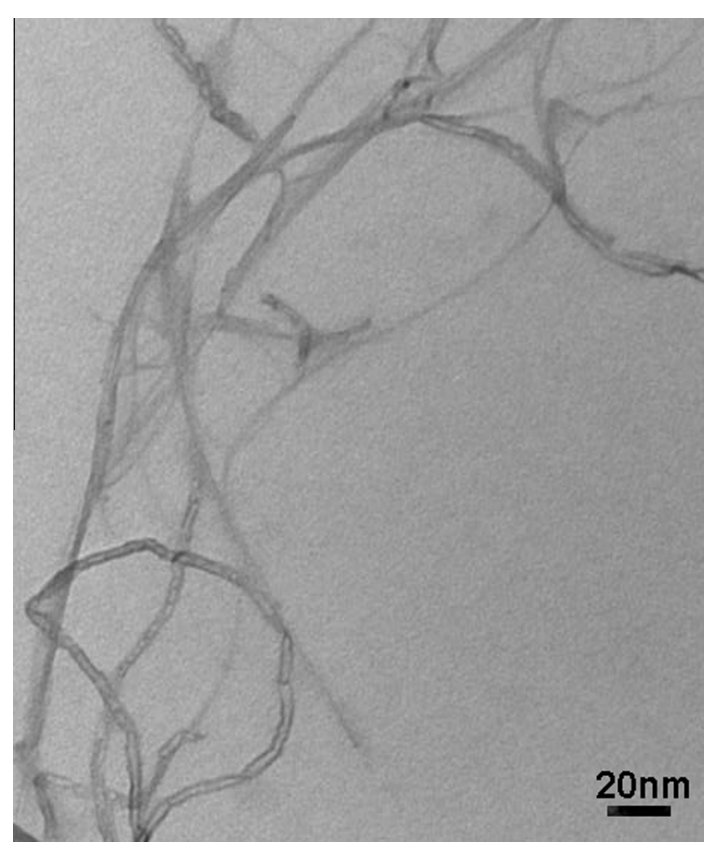

Fig. 1. Transmission electron microscopy image of the pluronic dispersed DWCNT sample used for instillation. 
staining, and two were frozen at $-80^{\circ} \mathrm{C}$ for subsequent immunofluorescence staining.

The following 23 cytokines and chemokines (IL-1a, IL-1b, IL-2, IL-3, IL-4, IL-5, IL-6, IL-9, IL-10, IL-12 (p40), IL-12 (p70), IL-13, IL17, CSF1/MCSF, CSF3/GCSF, IFN-g, CXCL1/KC, CCL2, CCL3, CCL4, CCL5, CCL11/Eotaxin and TNF-a) were quantified in the BAL fluid (BALF) and serum using Luminex technology (LINCOplex 23, Linco Research, St. Charles, MO) as described in [12]. Glutathione (GSH) and catalase activity levels in BALF were determined by high-performance liquid chromatography (Shimadzu, USA), as described in [29].

For immunofluorescence staining, cytospin slides were fixed with methanol at $-20^{\circ} \mathrm{C}$ for $10 \mathrm{~min}$ and with acetone $\left(-20^{\circ} \mathrm{C}\right)$ for $1 \mathrm{~min}$. The slides were washed twice in PBS and then blocked with PBS containing 0.1\% BSA for 10 min at room temperature, followed by draining. For (i) macrophage and (ii) granulocyte detection, slides were incubated with (i) rat anti-mouse Mac-3 antibody (BD Pharmingen) and (ii) goat anti-rat Ly6G/Ly6C (BD Pharmingen), in PBS containing 1\% BSA for $60 \mathrm{~min}$, and washed three times in PBS. This step was followed by incubation with the secondary antibodies Chromeo ${ }^{\mathrm{TM}} 642$ Goat anti-Mouse IgG (Active Motif) and Texas red (596 nm) anti-rat conjugate (Vector Labs) at the recommended dilution, in PBS containing 1\% BSA, for $30 \mathrm{~min}$. Subsequently, slides were washed three times with PBS. After staining cell nuclei by DAPI (Invitrogen), one drop of aqueous mounting medium was added on the cover slip and inverted carefully on a glass slide. Cell numbers and DWCNT agglomerate particles were counted using ImageJ (http://rsbweb.nih.gov/ij/) image processing analysis, according to the procedure described previously [30]. The black carbon particles in a bright field image were considered as DWCNT agglomerates. The bright field images were inverted into a dark field to allow for superposition with respective fluorescent images. To illustrate the uptake of DWCNT agglomerate particles, bright spots (carbon particles) were highlighted by green pseudocolour in the dark field, while the cytoplasma was shown by red fluorescent staining with anti-Mac-3 or anti-Ly6G/Ly6C. For quantification of DWCNT-particle laden cells, or more precisely, particle-associated cells, BAL macrophages and granulocytes were differentiated in pseudo-green particle free (negative) and pseudo-green particle associated (positive) cells.

All values are presented as mean \pm standard deviations. Significant differences between the two groups were evaluated by the Student's $t$-test or, between more groups, by one-way ANOVA. We performed regression analysis as well as Tukey's and Bonferroni's multiple comparison tests to identify the source of variance. Cytokine levels are given as concentration changes relative to sham, and for BALF levels, only changes over 10-fold (relative to the sham group) were defined as biological significant. Statistical analysis was performed with GraphPad Prism 5.0 (GraphPad Software, USA). Statistical significance is marked as follows: ${ }^{*} p<0.05$; ${ }^{* *} p<0.01 ;$ and ${ }^{* * *} p<0.001$.

\section{Results and discussion}

\subsection{DWCNT cause persistent alveolar inflammation}

Pharmaceutical functionalized DWCNT present a promising vehicle for intracellular drug targeting. On that aim, not pristine tubes as analysed here, but constructs with surface-functionalization, employing target specific probes, peptides or other ligands, are used to archive their pharmaceutical purpose. These pharmaceutical surface modifications will to a large extent also determine the acute fate and biocompatibility of the materials. However, once deposited in the lungs, and after a while in particular, the biopersistent CNT-backbone may remain as high burden in the alveolar region, where particle clearance is known to be poor (as reviewed by [31]), and pulmonary inflammation is a sensitive response to alveolar retained carbon nanoparticles with high surface area [11]. For that reason, we have decided to study the inflammatory response to pristine DWCNT in general, rather than that of a specific functionalization. On a related note, it may be mentioned that $\mathrm{COOH}$-functionalized SWCNTs have been shown to be even less biocompatible and more toxic than the corresponding pristine specimen [32-34].

In the present study, we show acute pulmonary inflammation in young and healthy mice upon intratracheal delivery of pluronic-dispersed DWCNT. This pulmonary response is characterised by a dynamic alveolar accumulation of neutrophil granulocytes on day 1 after instillation (Fig. 2) as assessed by bronchoalveolar lavage (BAL). During the first week after exposure, the number of neutrophilic BAL cells decreased from $239 \times 10 \mathrm{E} 3$ cells on day 1 , to $84 \times 10 \mathrm{E} 3$ on day 3 after instillation, but climbed back up to $150 \times 10 \mathrm{E} 3$ cells until day 7 . During the same time interval, the number of macrophages and lymphocytes increased continuously. As a result, the cellular composition of leucocytes, recovered by BAL, changed most substantially during the acute response, with $64 \%$ neutrophils on day 1, after DWCNT treatment. However, the fraction of neutrophils persisted at levels of $22 \%$ and $23 \%$ on day 3 and day 7 after treatment (Fig. 2). This lack of rapid resolution of the neutrophilic cell influx in the alveolar compartment of the lungs strongly indicated a sustained inflammatory response induced by DWCNTs. Another important indication of ineffective resolution and persistent inflammation was given by the accumulation of multinucleated macrophages, a crucial sign of foreign body reaction. Multinucleated macrophage numbers increased significantly from day 3 on. Moreover, the gradually increasing level of lymphocytes pointed to a disturbance of resolution of the acute response. BAL cell samples from the untreated control or sham group consisted almost exclusively of alveolar macrophages (95\%). Sham exposure caused no inflammatory response, but vehicle (1\% Pluronic F-127) instillation apparently enhanced the yield of BAL cell recovery about 2-fold, for macrophages, neutrophils and lymphocytes (Fig. 2). Instillation of the Pluronic F-127 detergent seems to increase the lavagability and recovery of lung leucocytes from the airspace, which is in accordance with its impact on cell adhesion which has been described for endothelial cells in vitro [35].

Upon inflammation, newly recruited macrophages and neutrophils present the front-line of early inflammatory responses against foreign materials in the lungs and accumulate in the airspace. In contrast to alveolar macrophages, neutrophils are short lived and, accordingly, their numbers decreased rapidly after day 1, whereas macrophages prevailed again from day 3 on. In fact, macrophage numbers increased threefold from day 1 to day 7 , as did the numbers of lymphocytes which had been close to the detection level during the initial state of inflammation.

The dynamic changes in alveolar leucocyte composition and numbers were accompanied by the release of a number proinflammatory cytokines, which were for IL-13, CSF3, CXCL1 and CCL3 found with over 100-fold increased concentrations in BAL fluid of DWCNT treated mice (BALF) (Fig. 3). The highest boost was detected for CSF3 (GCSF, granulocyte colony-stimulating factor), reaching concentrations over $3000 \mathrm{pg} / \mathrm{ml}$ at day 1 after DWCNT treatment (see Supplementary Table 1). However for the acute phase cytokines CSF3 and IL-6, BAL levels also declined again rapidly by day 7 . CCL3 in contrast remained 100 times elevated over the period of investigation, which matches with at day 7 still high neutrophil counts. CCL2, the major macrophage recruiting chemokine as well as Eotaxin, IFNg and IL-12 levels were characterised by over time increasing BAL concentrations which could 
BAL Cell Differentiation

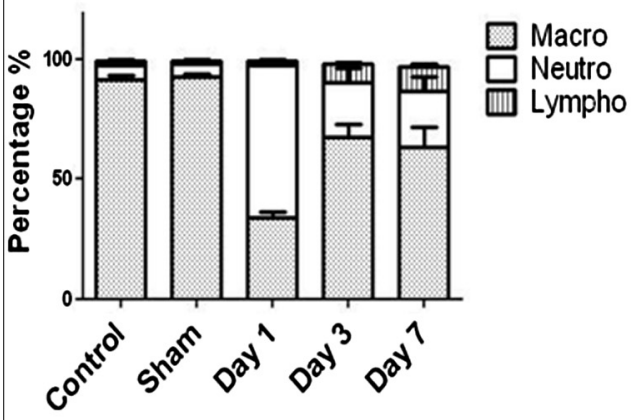

BAL Cell Differentiation
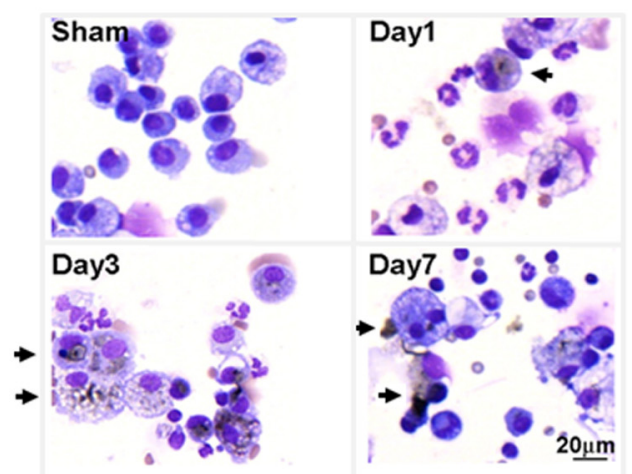

Macrophages

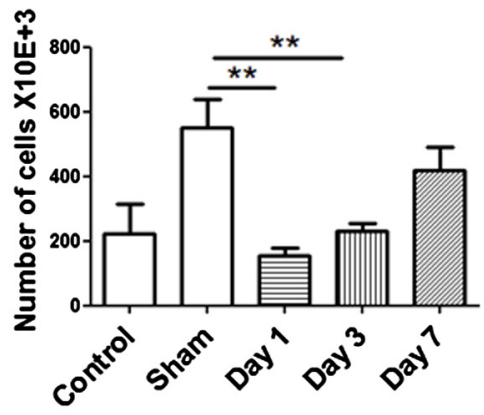

Neutrophils
Polynuclear Macrophages

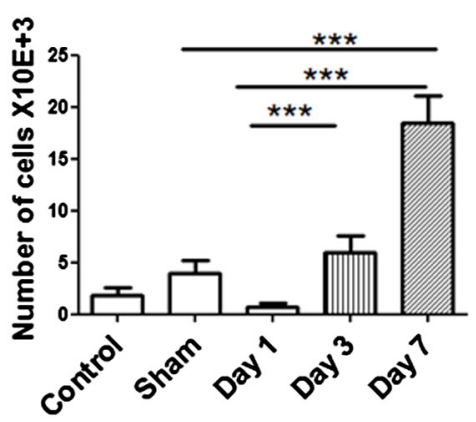

Lymphocytes
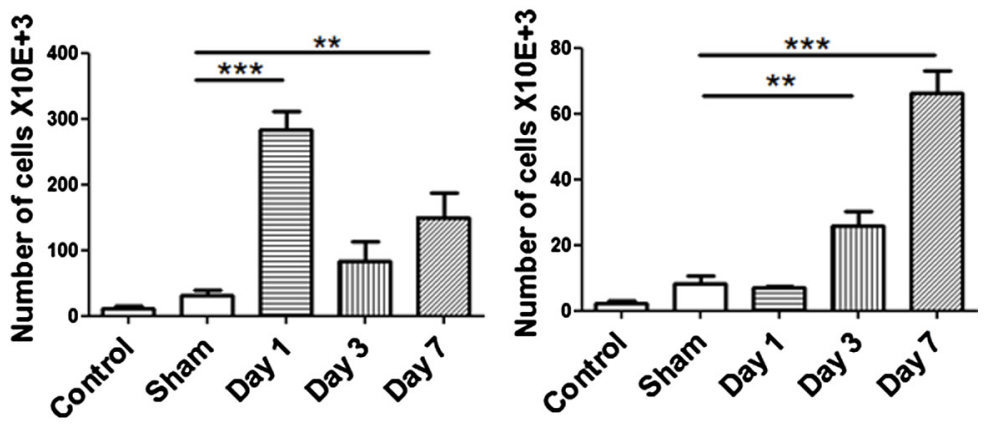

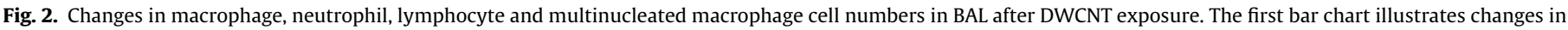

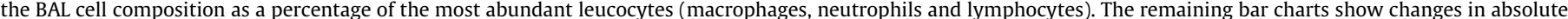

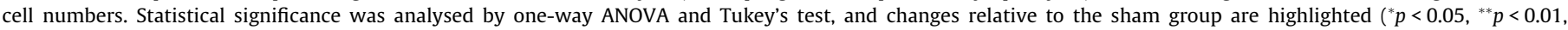

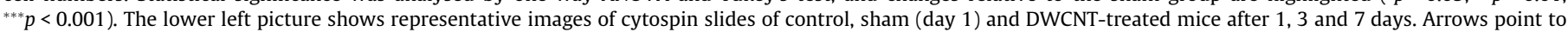
alveolar macrophages with visible DWCNT-agglomerate particles.

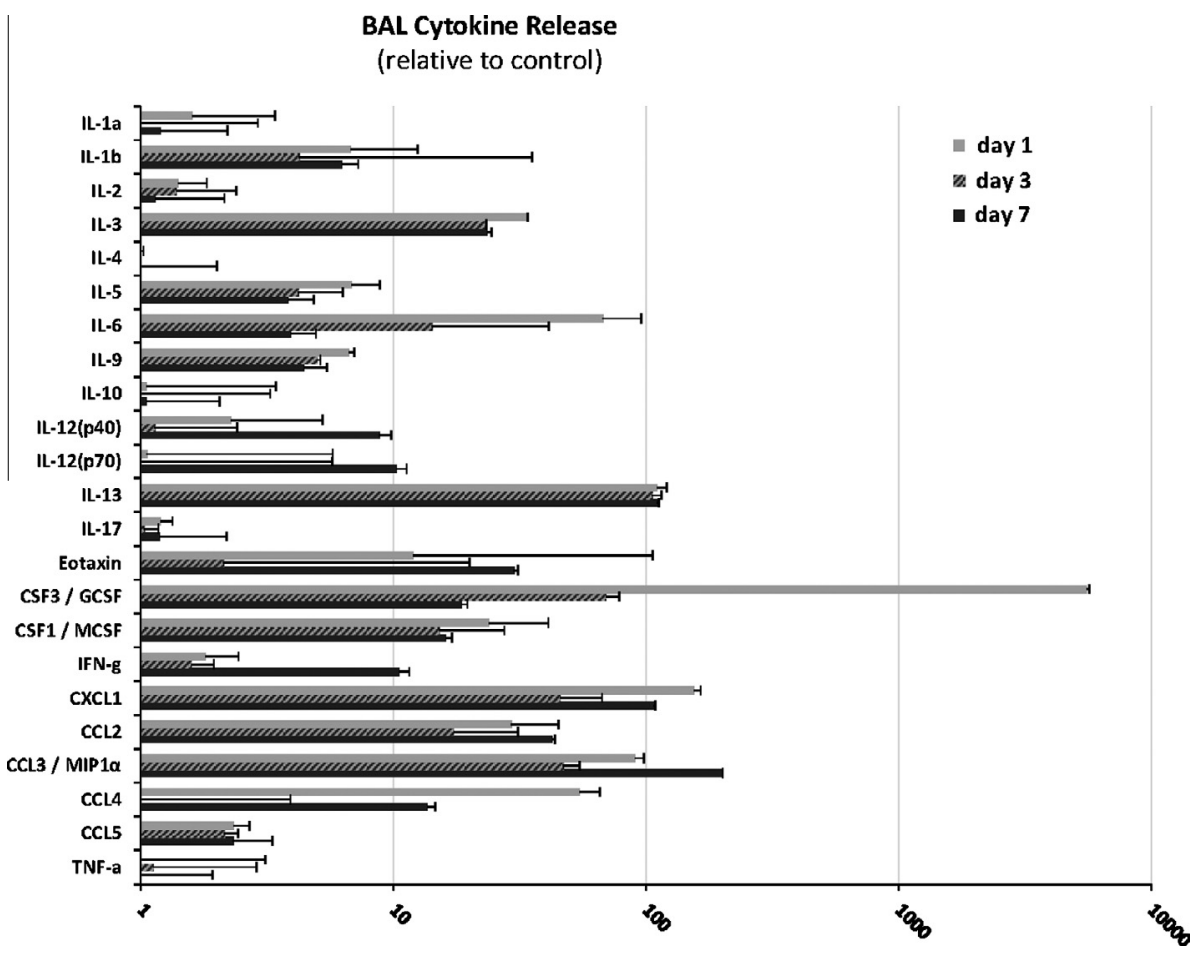

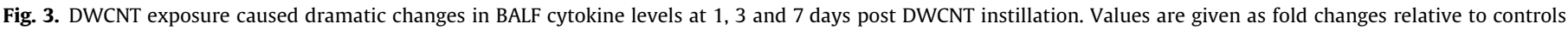
(+SEM) at the log scale. 


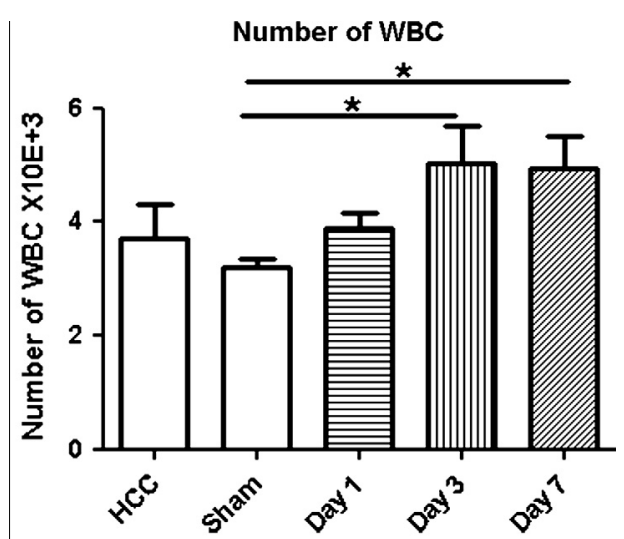

Neutrophils \%

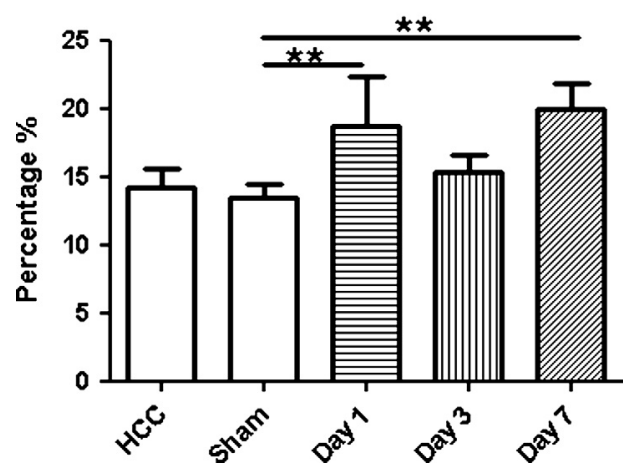

Fig. 4. DWCNT exposure caused a moderate yet significant increase in the white blood cell count (WBC) after 3 and 7 days, as well as an elevated fraction of neutrophil granulocytes in the peripheral blood of DWCNT-treated mice after 1 and 7 days. Statistical significance was analysed by one-way ANOVA and Tukey's test, and changes relative to the sham group are highlighted $\left({ }^{*} p<0.05,{ }^{* *} p<0.01\right)$.

Day 1
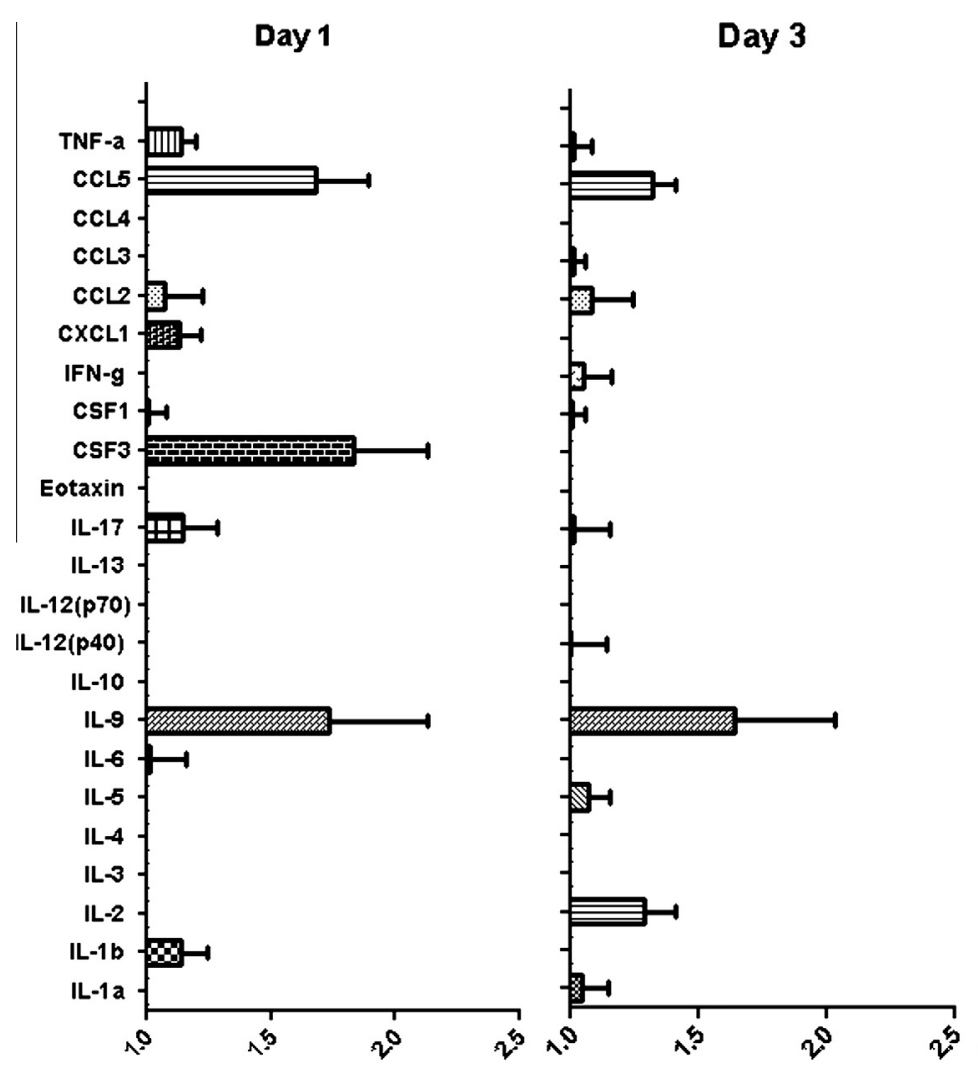

Day 3

Day 7 be associated with increased macrophage and leucocyte accumulation or even a rise in cell activation.

Overall, the biphasic cytokine response was observed, characterised by cytokines with initial high but over time decreasing levels but also those with highest levels at day 1 and 7 . This represents a pattern which matched well with the dynamics of the neutrophil cell numbers, which also decreased from day 1 to day 3, but increased again until day 7 (Fig. 2). In contrast to the mentioned Th1 and acute phase cytokines, the op to day 7still high Th2 cytokine levels of IL-13 and CCL11 (Eotaxin), with about 200 and $500 \mathrm{pg} / \mathrm{ml}$, might indicate long-term effects related to lung injury beyond the time of investigation. Accordingly, these cytokines have besides their well-known involvement in allergic inflammation also been connected to abnormal tissue regeneration as observed during pulmonary fibrosis [36]. In this context, Th2 lymphocyte derived IL-5, and IL13 might contribute to CNT-triggered pulmonary fibrosis.

\subsection{DWCNT elicit mild systemic inflammatory changes}

The peripheral white blood cell counts were elevated at day 3 and 7 but not day 1 after DWCNT treatment, indicating a moderate systemic inflammatory response in particular at the later phase (Fig. 4). In contrast, the percentage of neutrophils in the blood increased at day 1 and 7 only, but had returned to baseline at day 3 . On a related note, the granulocyte stimulating CSF3 was found two times elevated only at day 1, but CCL5 blood levels display a similar pattern as the neutrophil amount. Blood concentrations of the Tcell cytokine IL-9 revealed elevated over all time points relative to controls, whereas lymphocyte numbers remained constant (not shown). Over all, the blood cytokine response was much less expressed in the blood as compared to the lungs (Fig. 5). Though changes were comparatively moderate (1.5-2-fold), the pattern and course of up-down-up in the regulation of cytokines was

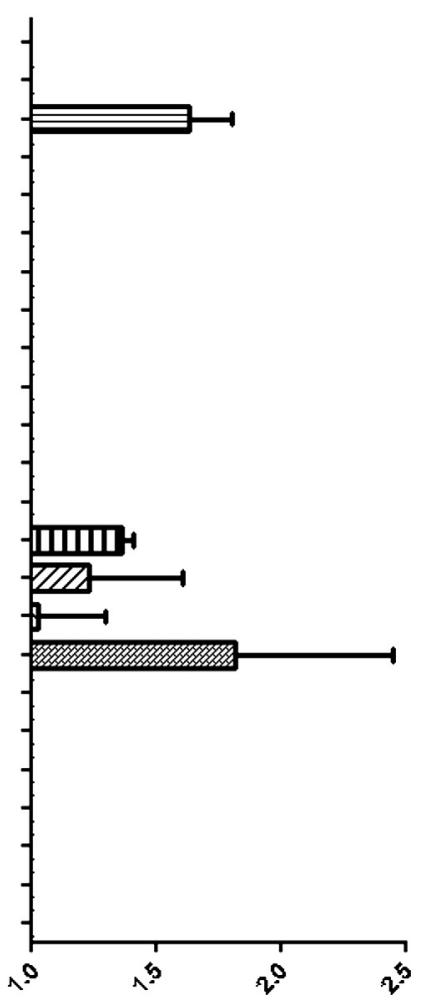

Fig. 5. Changes of serum cytokine levels at 1, 3 and 7 days post DWCNT instillation, relative to the sham-treated group. 
consistent between the different compartments. A direct communication between nanotubes challenged pulmonary tissue and the circulation has previously been described as by means of gene expression [18,37].

\subsection{DWCNT agglomerate uptake by alveolar macrophages but not by granulocytes}

Alveolar macrophages, as professional phagocytes and potent initiators of inflammation, have been extensively studied, with regard to their responses to various kinds of respirable particulate matter. In this context, 'frustrated phagocytosis' has been considered as the underlying mechanism of chronic toxicity of fibre-shaped materials, (e.g. asbestos or particular samples of multi-walled carbon nanotubes), which follow the classical fibre pathogenicity paradigm $[10,38]$. For less rigid tubes like single- and double-walled carbon nanotubes, as investigated in this study, a rather curled but not needle-shaped agglomerate structure was observed (Fig. 1). Therefore, the relevant pathway of toxicity is not clear yet.
To elucidate DWCNT cell interactions, we analysed the uptake of DWCNT agglomerates by the two cardinal professional phagocytes, the alveolar macrophages and, upon inflammation, invading neutrophils. Cell specific antibodies for macrophages, Mac3 (CD107b, LAMP2) and granulocytes, Ly6G/Ly6C (granulocyte receptor-1) were employed to differentiate macrophages or neutrophils on cytospin preparations of BAL cells by immunofluorescence. Quantification of BAL leucocytes by optical microscopy either associated with black CNT agglomerates or without visible particles was used as an indicator for the internalization of the mass of instilled carbon material. Cell and fluorescent densities were quantified, according to the procedure described [30]. As shown in Fig 6A-D, only macrophages but not neutrophils could be identified as internalizing CNT agglomerates (Fig. 6A-D). While the number of BAL macrophages increased from day 1 until day 7 after exposure (Fig. 2), the number of macrophages with engulfed DWCNT agglomerates remained rather constant (Fig. 6E), suggesting that phagocytosis was not limited by the number of macrophages.

The fact that Ly6G/Ly6C-positive neutrophils failed to effectively internalize particle agglomerates might be surprising since
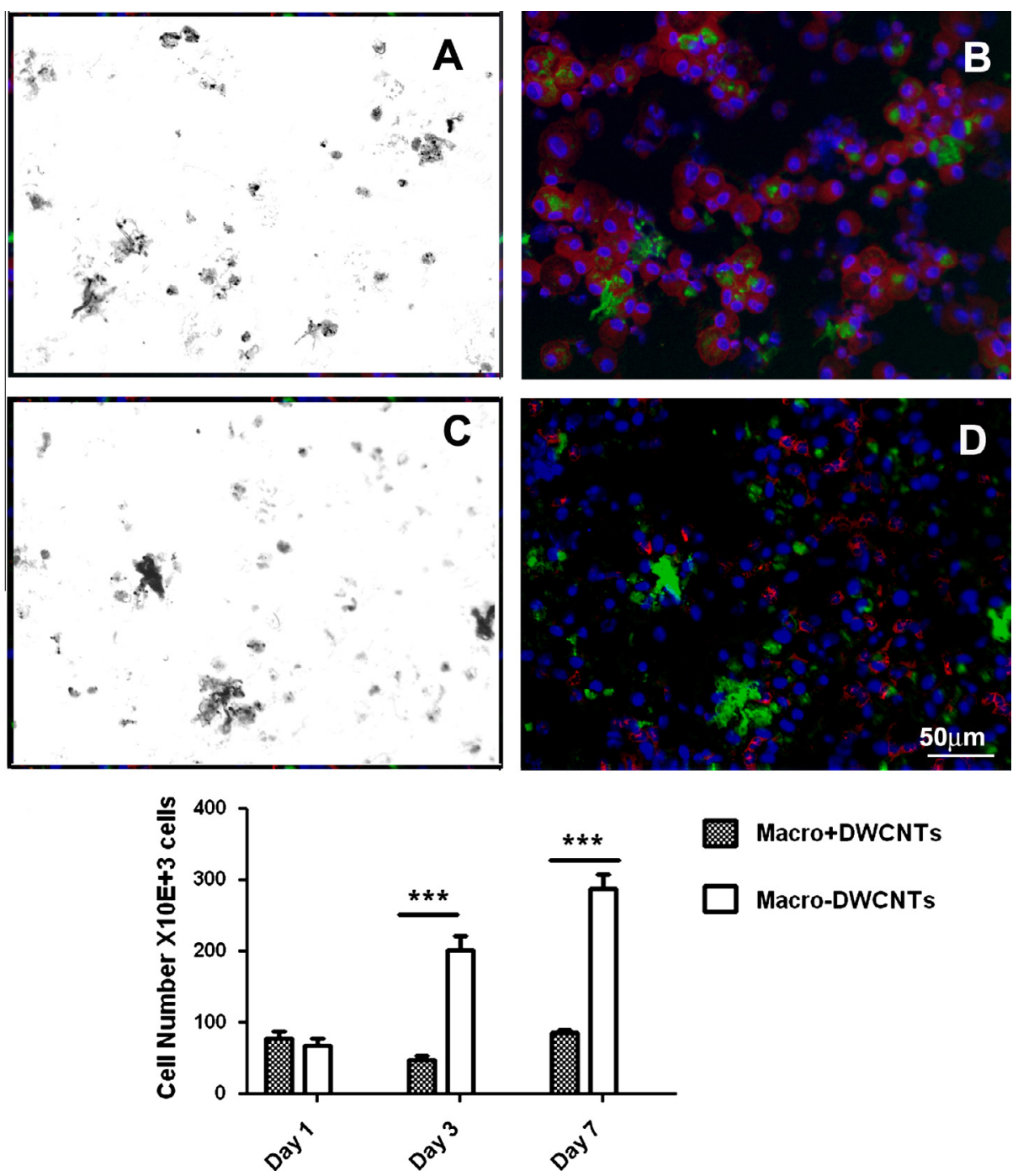

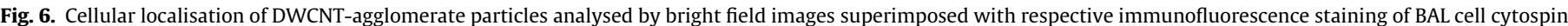

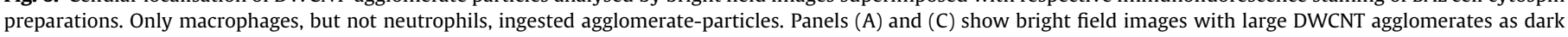

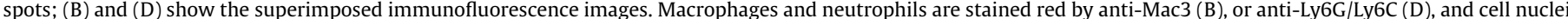

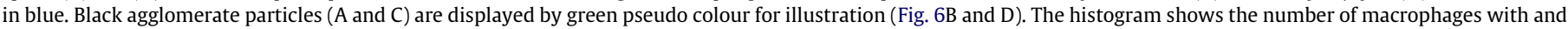

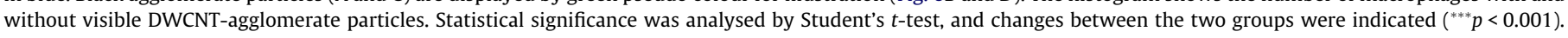




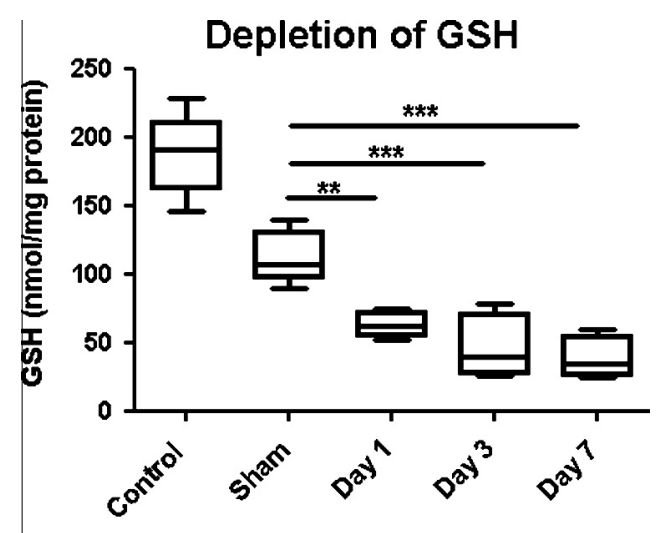

Catalase activity levels

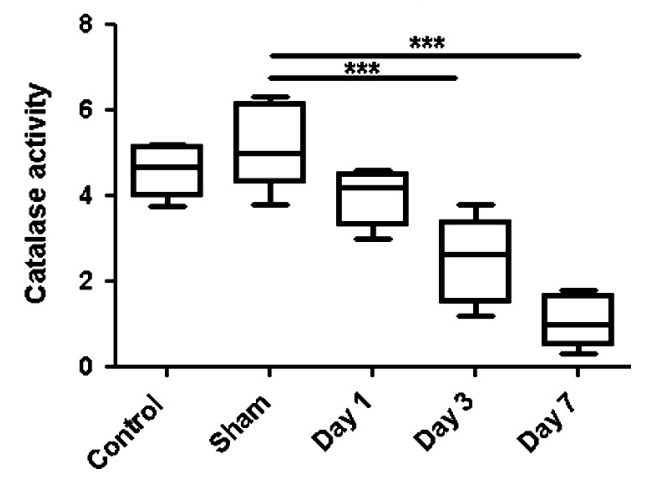

Fig. 7. DWCNT exposure caused a progressive depletion of glutathione (GSH) levels and catalase enzyme activity in lung tissue. Statistical significance was analysed compared to sham groups.

granulocytes are known to effectively phagocytose opsonized particles, and biodegradation of SWCNTs by neutrophils in vitro has been described [39]. Kagan and colleagues had already previously shown that degraded CNTs do not generate an inflammatory response when aspirated into the lungs of mice and thus suggest that the extent to which CNTs are biodegraded may be a major determinant of the scale and severity of the associated inflammatory responses in exposed individuals. To date, there is still no clear evidence that neutrophil granulocytes, despite recruited in large numbers to the site of CNT-injury, can effectively internalize the nanomaterial and thereby directly contribute to its degradation.
A recent study from Shvedova and colleagues showed the contribution of myeloperoxidase, a lysosomal enzyme most abundantly expressed in neutrophil granulocytes but to a lesser extent also in certain macrophages, to oxidative biodegradation of SWCNT [40]. However, also in their study, no significant uptake of SWCNT-agglomerates can be detected in neutrophils but only in macrophages. It might be noteworthy to mention that in all of our various particle instillation studies, we have never observed any recognisable amounts of carbon particles associated with granulocytes [12,41-43].

Since nanoparticles, in general, have been suggested to express their pro-inflammatory toxicity via their oxidative properties, and to further characterise the kind of pulmonary injury, we quantified lung glutathione antioxidant levels as well as the activity of the antioxidant enzyme catalase. As illustrated in Fig. 7, DWCNT exposure significantly reduced both endogenous antioxidant defence systems, with the depletion even increasing over time and resulting in minimal levels on day 7. Pulmonary deposited carbonaceous nanoparticles have been described as causing oxidative stress to the lungs, which has been further related to the inflammatory response caused by these materials [44]. So far, there have been only few studies that have focused on the respiratory toxicity of DWCNT. Our findings may even contradict the current literature regarding the involvement of oxidative stress resulting from DWCNT-caused injury. In this context, Meunier and colleagues described a ROS independent, but DWCNTs phagocytosis-dependent cause of DWCNT triggered inflammation via a cascade of a toll-like receptor and Nlrp3 inflammasome activation, leading to IL-1 $\beta$ release in human monocytes [45]. Crouzier et al., on the other hand, investigated oxidative perturbations using ESR spin trapping experiments of DWCNT-challenged lungs [46]. In their model, the authors showed clear signs demonstrating pulmonary and systemic acute inflammatory reactions, which were accompanied by a decrease in local oxidative stress levels. One major difference between these two studies and ours is that we have looked at later time points that exceeded the acute response to get insight into the potential malfunction of a resolution for pulmonary inflammation. Another more comprehensive study, which looked into lung inflammation, fibrosis, oxidative stress and mutagenesis in the lungs of mice after multi-day exposure to SWCNTs, also supports our finding of exposure-related depletion of the antioxidant system [26]. Metal impurities about 20\%, in particular iron and nickel levels of CNTs, as remain from their synthesis, have been shown to trigger nanotube exposure related oxidative stress in vitro and in vivo [22,47]. According to our measurements however, the

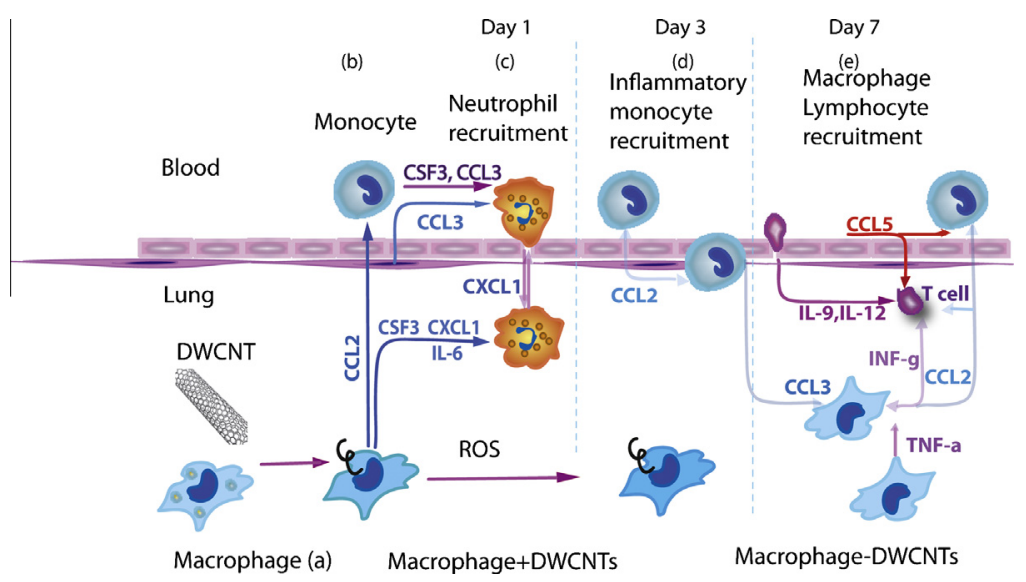

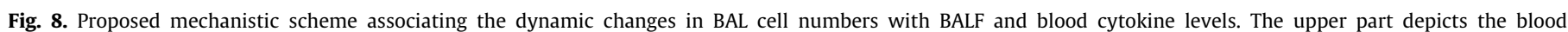

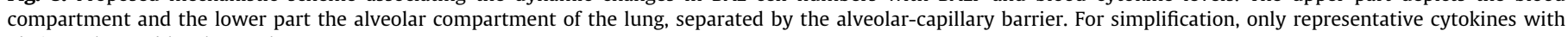
obvious changed levels are shown. 
DWCNT content of $2.45 \% \mathrm{Fe}$ and $0.02 \% \mathrm{Ni}$ can hardly explain the overtime increasing oxidative stress response as illustrated in fig. 7. We therefore rather assume that the persistent inflammatory response described here is the main driver of the exhaustion of the local antioxidant system, by inflammatory radical generating enzymes such as monoxigenases, NADPH oxidases or peroxidases, which are produced and released by inflammatory cells.

\section{Proposed scheme of inflammatory response}

Our results can be summarised in a schematic illustration of the dynamic interplay of the different cell types and cytokines during the course of DWCNT-triggered pulmonary inflammation (Fig. 8). This figure depicts two parallel, horizontal scenarios, in which the upper part shows the circulation and the lower part represents the alveolar compartment of the lung. During the early phase after alveolar DWCNT deposition, resident alveolar macrophages attempt to phagocytose and, thereby, clear the particulate 'invader', which gives rise to macrophage activation with subsequent cytokine and sustained ROS release (Fig. 8a). Concurrently, cytokines (such as CCL2, CCL3, IL6 and CSF3) released by macrophages induce blood monocytes to secrete CSF3 (Fig. 8b) and thus, ultimately, amplify the pro-inflammatory response by augmentation and attraction of granulocytes (day 1, Fig. 8c). The increasing production of CCL2 and CSF3 chemokines further induces monocyte recruitment to the lungs, accompanied by subsequent maturation of macrophages. Increasing macrophage numbers coincide with the influx of to the alveoli attracted peripheral leucocytes (Fig. 8) and correspondingly newly recruited macrophages, without obvious CNTsinclusion accumulate in the lungs. Increased numbers of by inflammatory cytokines activated monocytes/macrophages and granulocytes may now also contribute to the temporal reduction of cytokine levels by cytokine absorption in the lung and blood on day 3 (Fig. 8d). From day 3 to day 7, lymphocyte activation and attraction by IL-9,-12 and CCL5 gather momentum and, accordingly, lymphocytes accumulate in the alveolar region. By day 7 , in the alveolar compartment and circulation, a self-perpetuating response must apparently have developed. New macrophages stimulate lymphocytes and granulocytes to maintain a pro-inflammatory cytokine response (Fig. 8). Cytokine profiles for IL-9 and CCL5 are systemically elevated and support the recruitment of new lymphocytes and monocytes (Fig. 8e). As result, and after 1 week of DWCNT retention, still no effective resolution of pulmonary inflammation could be archived which divines the formation of local, but chronic inflammation. This assumption is supported by the development of multinucleated and giant macrophages (Fig. 2), which indicate a failure of particle clearance. In contrast, we have shown in previous instillation studies, with similar dosing but spherical carbon nanoparticles, that the respective acute pulmonary inflammatory response is completely resolved within 7 days at the cellular and molecular level [10]. Notwithstanding that even in the latter case, considerable amounts of the carbon nanoparticle agglomerates remain in the lungs as well but do not induce giant cell formation. Future experiments with prolonged investigation periods should examine these processes in more detail. In a related context, we have previously described the inflammatory reaction in responses to pulmonary application of PEI-based siRNA nanocarriers as an alarming signal with regard to the biocompatibility of the respective material for pulmonary drug delivery [48]. Similarly, we think that our DWCNT results might caution as well against the use of this biopersistent material for pulmonary drug delivery.

In summary, we have shown that intratracheal exposure to a dose of $50 \mu \mathrm{g}$ pluronic-dispersed, pristine DWCNT for each mouse causes an acute inflammatory response in the lungs and minor impairment in the blood compartment, which does not resolve within 1 week after exposure but is accompanied by a significant and over time aggravating depletion of the pulmonary antioxidant system. DWCNT agglomerates were phagocytosed by macrophages but not neutrophil granulocytes, thereby rendering the impact of the latter on clearance or degradation implausible. In this context, it needs to be noted that the instilled DWCNT suspension consists of large agglomerates besides individually dispersed CNTs. This morphology might be seen as a worst case scenario for either the pulmonary application of inadequately dispersed, or after inhalation in the lung agglomerated constructs, which must be avoided in therapeutic approaches.

\section{Acknowledgements}

This work is partially supported by the European Commission's 7th Framework Programme, through projects HEALTH-2007-2.4.17 Contract No. 201587 (ANTICARB). Support from the German Research Foundation (DFG) Research Grant TI (Furong Tian) 696/1-1 through projects NanoSci-ERA Nanotruck is also acknowledged.

\section{Appendix A. Supplementary material}

Supplementary data associated with this article can be found, in the online version, at http://dx.doi.org/10.1016/j.ejpb.2013.03.008.

\section{References}

[1] C. Klumpp, K. Kostarelos, M. Prato, A. Bianco, Functionalized carbon nanotubes as emerging nanovectors for the delivery of therapeutics, Biochimica et Biophysica Acta 1758 (2006) 404-412.

[2] A. Antonelli, S. Serafini, M. Menotta, C. Sfara, F. Pierige, L. Giorgi, G. Ambrosi, L. Rossi, M. Magnani, Improved cellular uptake of functionalized single-walled carbon nanotubes, Nanotechnology 21 (2010) 425101.

[3] S. Kraszewski, A. Bianco, M. Tarek, C. Ramseyer, Insertion of short aminofunctionalized single-walled carbon nanotubes into phospholipid bilayer occurs by passive diffusion, PLoS ONE 7 (2012) e40703.

[4] N.W. Shi Kam, T.C. Jessop, P.A. Wender, H. Dai, Nanotube molecular transporters: internalization of carbon nanotube-protein conjugates into Mammalian cells, Journal of the American Chemical Society 126 (2004) 6850-6851.

[5] E. Venturelli, C. Fabbro, O. Chaloin, C. Menard-Moyon, C.R. Smulski, T. Da Ros, K. Kostarelos, M. Prato, A. Bianco, Antibody covalent immobilization on carbon nanotubes and assessment of antigen binding, Small (Weinheim an der Bergstrasse, Germany) 7 (2011) 2179-2187.

[6] M. Pacurari, V. Castranova, V. Vallyathan, Single- and multi-wall carbon nanotubes versus asbestos: are the carbon nanotubes a new health risk to humans?, Journal of Toxicology and Environmental Health, Part A 73 (2010) 378-395

[7] K. Donaldson, C. Poland, Inhaled nanoparticles and lung cancer - what we can learn from conventional particle toxicology, Swiss Medical Weekly 142 (2012).

[8] K. Donaldson, F. Murphy, A. Schinwald, R. Duffin, C.A. Poland, Identifying the pulmonary hazard of high aspect ratio nanoparticles to enable their safety-bydesign, Nanomedicine (London) 6 (2011) 143-156.

[9] K. Donaldson, C.A. Poland, Nanotoxicology: new insights into nanotubes, Nature Nanotechnology 4 (2009) 708-710.

[10] K. Ganguly, S. Upadhyay, M. Irmler, S. Takenaka, K. Pukelsheim, J. Beckers, E. Hamelmann, H. Schulz, T. Stoeger, Pathway focused protein profiling indicates differential function for IL-1B, -18 and VEGF during initiation and resolution of lung inflammation evoked by carbon nanoparticle exposure in mice, Particle and Fibre Toxicology 6 (2009) 31.

[11] T. Stoeger, C. Reinhard, S. Takenaka, A. Schroeppel, E. Karg, B. Ritter, J. Heyder, H. Schulz, Instillation of six different ultrafine carbon particles indicates a surface area threshold dose for acute lung inflammation in mice, Environmental Health Perspectives 114 (2006) 328-333.

[12] A.A. Gotz, A. Vidal-Puig, H.G. Rodel, M.H. de Angelis, T. Stoeger, Carbonnanoparticle-triggered acute lung inflammation and its resolution are not altered in PPARgamma-defective (P465L) mice, Particle and Fibre Toxicology 8 (2011) 28.

[13] G. Oberdorster, E. Oberdorster, J. Oberdorster, Nanotoxicology: an emerging discipline evolving from studies of ultrafine particles, Environmental Health Perspectives 113 (2005) 823-839.

[14] F. Tian, D. Cui, H. Schwarz, G.G. Estrada, H. Kobayashi, Cytotoxicity of singlewall carbon nanotubes on human fibroblasts, Toxicology in vitro: An International Journal Published in Association with BIBRA 20 (2006) 12021212.

[15] D. Cui, F. Tian, C.S. Ozkan, M. Wang, H. Gao, Effect of single wall carbon nanotubes on human HEK293 cells, Toxicology Letters 155 (2005) 73-85. 
[16] X. Zhao, R. Liu, Recent progress and perspectives on the toxicity of carbon nanotubes at organism, organ, cell, and biomacromolecule levels, Environment International 40 (2012) 244-255.

[17] J.B. Mangum, E.A. Turpin, A. Antao-Menezes, M.F. Cesta, E. Bermudez, J.C. Bonner, Single-walled carbon nanotube (SWCNT)-induced interstitial fibrosis in the lungs of rats is associated with increased levels of PDGF mRNA and the formation of unique intercellular carbon structures that bridge alveolar macrophages in situ, Particle and Fibre Toxicology 3 (2006) 15.

[18] A.A. Shvedova, J.P. Fabisiak, E.R. Kisin, A.R. Murray, J.R. Roberts, Y.Y. Tyurina, J.M. Antonini, W.H. Feng, C. Kommineni, J. Reynolds, A. Barchowsky, V. Castranova, V.E. Kagan, Sequential exposure to carbon nanotubes and bacteria enhances pulmonary inflammation and infectivity, American Journal of Respiratory Cell and Molecular Biology 38 (2008) 579-590.

[19] H. Nagai, Y. Okazaki, S.H. Chew, N. Misawa, Y. Yamashita, S. Akatsuka, T. Ishihara, K. Yamashita, Y. Yoshikawa, H. Yasui, L. Jiang, H. Ohara, T. Takahashi, G. Ichihara, K. Kostarelos, Y. Miyata, H. Shinohara, S. Toyokuni, Diameter, rigidity of multiwalled carbon nanotubes are critical factors in mesothelial injury, carcinogenesis, Proceedings of the National Academy of Sciences of the United States of America 108 (2011) E1330-E1338.

[20] J. Palomaki, E. Valimaki, J. Sund, M. Vippola, P.A. Clausen, K.A. Jensen, K. Savolainen, S. Matikainen, H. Alenius, Long, needle-like carbon nanotubes and asbestos activate the NLRP3 inflammasome through a similar mechanism, ACS Nano 5 (2011) 6861-6870.

[21] A. Schinwald, F. Murphy, A. Prina-Mello, C. Poland, F. Byrne, J. Glass, J. Dickerson, D. Schultz, D. Movia, C. Jeffree, W. Macnee, K. Donaldson, The threshold length for fibre-induced acute pleural inflammation: shedding light on the early events in asbestos-induced mesothelioma, Toxicological Sciences: An Official Journal of the Society of Toxicology (2012).

[22] H.J. Johnston, G.R. Hutchison, F.M. Christensen, S. Peters, S. Hankin, K. Aschberger, V. Stone, A critical review of the biological mechanisms underlying the in vivo and in vitro toxicity of carbon nanotubes: the contribution of physico-chemical characteristics, Nanotoxicology 4 (2010) 207-246.

[23] F.A. Murphy, A. Schinwald, C.A. Poland, K. Donaldson, The mechanism of pleural inflammation by long carbon nanotubes: interaction of long fibres with macrophages stimulates them to amplify pro-inflammatory responses in mesothelial cells, Particle and Fibre Toxicology 9 (2012) 8.

[24] J.P. Ryman-Rasmussen, E.W. Tewksbury, O.R. Moss, M.F. Cesta, B.A. Wong, J.C. Bonner, Inhaled multiwalled carbon nanotubes potentiate airway fibrosis in murine allergic asthma, American Journal of Respiratory Cell and Molecular Biology 40 (2009) 349-358.

[25] K. Donaldson, F.A. Murphy, R. Duffin, C.A. Poland, Asbestos, carbon nanotubes and the pleural mesothelium: a review of the hypothesis regarding the role of long fibre retention in the parietal pleura, inflammation and mesothelioma, Part Fibre Toxicol 7 (2010) 5.

[26] A.A. Shvedova, E. Kisin, A.R. Murray, V.J. Johnson, O. Gorelik, S. Arepalli, A.F. Hubbs, R.R. Mercer, P. Keohavong, N. Sussman, J. Jin, J. Yin, S. Stone, B.T. Chen, G. Deye, A. Maynard, V. Castranova, P.A. Baron, V.E. Kagan, Inhalation vs. aspiration of single-walled carbon nanotubes in C57BL/6 mice: inflammation, fibrosis, oxidative stress, and mutagenesis 295 (2008) L552-L565.

[27] M.C. Jaurand, A. Renier, J. Daubriac, Mesothelioma: do asbestos and carbon nanotubes pose the same health risk?, Particle and Fibre Toxicology 6 (2009) 16

[28] C. Fabbro, H. Ali-Boucetta, T. Da Ros, K. Kostarelos, A. Bianco, M. Prato Targeting carbon nanotubes against cancer, Chemical Communications (Cambridge, England) 48 (2012) 3911-3926.

[29] A. Banerjee, M.B. Trueblood, X. Zhang, K.R. Manda, P. Lobo, P.D. Whitefield, D.E Hagen, N. Ercal, N-acetylcysteineamide (NACA) prevents inflammation and oxidative stress in animals exposed to diesel engine exhaust, Toxicology Letters 187 (2009) 187-193.

[30] F. Tian, A. Prina-Mello, G. Estrada, A. Beyerle, W. Moeller, H. Schulz, W. Kreyling, T. Stoeger, A novel assay for the quantification of internalized nanoparticles in macrophages, Nanotoxicology (2008) 232-242.

[31] W.G. Kreyling, M. Semmler-Behnke, S. Takenaka, W. Moller, Differences in the biokinetics of inhaled nano- versus micrometer-sized particles, Accounts of Chemical Research (2012).
[32] H.L. Liu, Y.L. Zhang, N. Yang, Y.X. Zhang, X.Q. Liu, C.G. Li, Y. Zhao, Y.G. Wang, G.G. Zhang, P. Yang, F. Guo, Y. Sun, C.Y. Jiang, A functionalized single-walled carbon nanotube-induced autophagic cell death in human lung cells through Akt-TSC2-mTOR signaling, Cell Death \& Disease 2 (2011) e159.

[33] S. Sachar, R.K. Saxena, Cytotoxic effect of poly-dispersed single walled carbon nanotubes on erythrocytes in vitro and in vivo, PLoS ONE 6 (2011) e22032.

[34] H. Tong, J.K. McGee, R.K. Saxena, U.P. Kodavanti, R.B. Devlin, M.I. Gilmour Influence of acid functionalization on the cardiopulmonary toxicity of carbon nanotubes and carbon black particles in mice, Toxicology and Applied Pharmacology 239 (2009) 224-232.

[35] J.L. Tan, W. Liu, C.M. Nelson, S. Raghavan, C.S. Chen, Simple approach to micropattern cells on common culture substrates by tuning substrate wettability, Tissue Engineering 10 (2004) 865-872.

[36] T.A. Wynn, Fibrotic disease and the $\mathrm{T}(\mathrm{H}) 1 / \mathrm{T}(\mathrm{H}) 2$ paradigm, Nature Reviews Immunology 4 (2004) 583-594.

[37] A. Erdely, T. Hulderman, R. Salmen, A. Liston, P.C. Zeidler-Erdely, D. SchweglerBerry, V. Castranova, S. Koyama, Y.A. Kim, M. Endo, P.P. Simeonova, Cross-talk between lung and systemic circulation during carbon nanotube respiratory exposure. Potential biomarkers, Nano Letters 9 (2009) 36-43.

[38] K. Donaldson, F.A. Murphy, R. Duffin, C.A. Poland, Asbestos, carbon nanotubes and the pleural mesothelium: a review of the hypothesis regarding the role of long fibre retention in the parietal pleura, inflammation and mesothelioma, Particle and Fibre Toxicology 7 (2010) 5.

[39] V.E. Kagan, N.V. Konduru, W. Feng, B.L. Allen, J. Conroy, Y. Volkov, Vlasova II, N.A. Belikova, N. Yanamala, A. Kapralov, Y.Y. Tyurina, J. Shi, E.R. Kisin, A.R Murray, J. Franks, D. Stolz, P. Gou, J. Klein-Seetharaman, B. Fadeel, A. Star, A.A Shvedova, Carbon nanotubes degraded by neutrophil myeloperoxidase induce less pulmonary inflammation, Nature Nanotechnology 5 (2010) 354-359.

[40] A.A. Shvedova, A.A. Kapralov, W.H. Feng, E.R. Kisin, A.R. Murray, R.R. Mercer C.M. St Croix, M.A. Lang, S.C. Watkins, N.V. Konduru, B.L. Allen, J. Conroy, G.P. Kotchey, B.M. Mohamed, A.D. Meade, Y. Volkov, A. Star, B. Fadeel, V.E. Kagan, Impaired clearance and enhanced pulmonary inflammatory/fibrotic response to carbon nanotubes in myeloperoxidase-deficient mice, PLoS ONE 7 (2012) e30923.

[41] K. Ganguly, S. Upadhyay, M. Irmler, S. Takenaka, K. Pukelsheim, J. Beckers, M.H. De Angelis, E. Hamelmann, T. Stoeger, H. Schulz, Impaired resolution of inflammatory response in the lungs of JF1/Msf mice following carbon nanoparticle instillation, Respiratory Research 12 (2011) 94.

[42] A.A. Gotz, J. Rozman, H.G. Rodel, H. Fuchs, V. Gailus-Durner, M. Hrabe de Angelis, M. Klingenspor, T. Stoeger, Comparison of particle-exposure triggered pulmonary and systemic inflammation in mice fed with three different diets, Particle and Fibre Toxicology 8 (2011) 30.

[43] A. Khandoga, T. Stoeger, A.G. Khandoga, P. Bihari, E. Karg, D. Ettehadieh, S Lakatos, J. Fent, H. Schulz, F. Krombach, Platelet adhesion and fibrinogen deposition in murine microvessels upon inhalation of nanosized carbon particles, Journal of Thrombosis and Haemostasis: JTH 8 (2010) 1632-1640.

44] T. Stoeger, S. Takenaka, B. Frankenberger, B. Ritter, E. Karg K. Maier, H. Schulz, O. Schmid, Deducing in vivo toxicity of combustion-derived nanoparticles from a cell-free oxidative potency assay and metabolic activation of organic compounds, Environmental Health Perspectives 117 (2009) 54-60.

[45] E. Meunier, A. Coste, D. Olagnier, H. Authier, L. Lefevre, C. Dardenne, J. Bernad, M. Beraud, E. Flahaut, B. Pipy, Double-walled carbon nanotubes trigger IL1 beta release in human monocytes through Nlrp3 inflammasome activation, Nanomedicine: Nanotechnology, Biology, and Medicine (2011).

[46] D. Crouzier, S. Follot, E. Gentilhomme, E. Flahaut, R. Arnaud, V. Dabouis, C. Castellarin, J.C. Debouzy, Carbon nanotubes induce inflammation but decrease the production of reactive oxygen species in lung, Toxicology 272 (2010) 3945.

[47] V.E. Kagan, Y.Y. Tyurina, V.A. Tyurin, N.V. Konduru, A.I. Potapovich, A.N Osipov, E.R. Kisin, D. Schwegler-Berry, R. Mercer, V. Castranova, A.A. Shvedova, Direct and indirect effects of single walled carbon nanotubes on RAW 264.7 macrophages: role of iron, Toxicology Letters 165 (2006) 88-100.

[48] A. Beyerle, A. Braun, A. Banerjee, N. Ercal, O. Eickelberg, T.H. Kissel, T. Stoeger Inflammatory responses to pulmonary application of PEI-based siRNA nanocarriers in mice, Biomaterials 32 (2011) 8694-8701. 